\title{
86. Products of Compact Fréchet Spaces
}

\author{
By Ken-ichi Tamano \\ Faculty of Liberal Arts, Shizuoka University \\ (Communicated by Kôsaku YosidA, M. J. A., Oct. 13, 1986)
}

1. Introduction. A topological space $X$ is called Fréchet if each point in the closure of a subset $A \subset X$ is the limit of a sequence from $A$. In 1972, E. Michael [5] raised the question whether there exist two compact Hausdorff spaces $X$ and $Y$ such that the product space $X \times Y$ is not Fréchet. After then, various examples of such spaces were constructed by T. K. Boehme and M. Rosenfeld [1] (under the continuum hypothesis $\mathrm{CH}$ ), V. I. Malyhin and B. E. Šapirovskiǐ [4], R. C. Olson [7] (under Martin's axiom), and P. Simon [8] (without extra set-theoretic assumptions).

Generalizing E. Michael's question for more than two compact spaces, T. Nogura [6] asked: For $n \geqq 2$, is there a compact Fréchet space $X$ such that $X^{n}$ is Fréchet but $X^{n+1}$ is not Fréchet?

The purpose of this paper is to answer the question positively under Martin's axiom. Indeed, using G. Gruenhage's technique [2] and Franklin compact spaces, we construct for each $n$ with $3 \leqq n \leqq \omega$, a compact Fréchet space $X$ such that $X^{k}$ is Fréchet for any $k<n$, but $X^{n}$ is not Fréchet.

All spaces are assumed to be Hausdorff. The symbol $\omega$ denotes the first infinite cardinal and, simultaneously, the set of non-negative integer's with the discrete topology.

2. Preliminaries. The Stone-Čech compactification of the countable discrete space $\omega$ is denoted by $\beta \omega$. For each $A \subset \omega$, the set $A^{*}$ is defined by $A^{*}=\operatorname{cl}_{\beta \omega} A-A$. Let $\mathscr{P}$ be an infinite family of disjoint clopen subsets of $\omega^{*}$. The Franklin compact space $F(\mathscr{P})$ is a quotient space of $\beta \omega$ obtained by the decomposition of $\beta \omega$ into $\left\{\omega^{*}-\cup \mathscr{Q}\right\}$, elements of $\mathscr{P}$, and one-point sets $\{n\}$ with $n \in \omega$. Express $F(\mathscr{P})$ as $\{\infty\} \cup \mathscr{Q} \cup \omega$, or more precisely, as $\left\{\infty_{\mathscr{P}}\right\} \cup \mathscr{P} \cup \omega$.

Note that every family $\mathscr{Q}$ of disjoint clopen subsets of $\omega^{*}$ can be written as $\mathscr{Q}=\left\{I^{*}: I \in \mathcal{G}\right\}$, where the family $\mathcal{G}$ is an almost disjoint family of infinite subsets of $\omega$. A family $\mathcal{G}$ is said to be almost disjoint if $I \cap J$ is finite for any distinct members $I, J \in \mathcal{G}$.

It is easy to check the following lemma :

Lemma 1. (a) $F(\mathscr{P})$ is a compact Hausdorff space.

(b) Each point of $\omega$ is isolated.

(c) Let $P \in \mathscr{Q}$ and $P=I^{*}$, where $I \in \mathcal{G}$. Then the family $\{\{P\} \cup(I-F)$ : $F$ is a finite subset of $\omega\}$ is a neighborhood base at the point $\{P\}$.

(d) The family $\left\{\left\{\infty_{\mathscr{Q}}\right\} \cup(\mathcal{P}-Q) \cup(\omega-\cup Q-F): Q\right.$ is a finite subfamily of $\mathcal{Q}$, and $F$ is a finite subset of $\omega\}$ is a neighborhood base at the point $\infty_{\mathscr{P}}$. 
The following lemma was essentially proved by T. K. Boehme and M. Rosenfeld [1], and V. I. Malyhin and B. E. Šapirovskiř [4]. G. Gruenhage [2] proved the case $n=\omega$.

Lemma 2. Let $1 \leqq n \leqq \omega$. Suppose that $\mathscr{P}=\bigcup\left\{\mathscr{Q}_{i}: i<n\right\}$ is an infinite maximal disjoint family of clopen subsets of $\omega^{*}$ such that $\mathcal{Q}_{i} \cap \mathscr{Q}_{j}=\varnothing$ whenever $i \neq j$. Then the product space $\prod\left\{\left\{\infty_{F\left(\mathscr{P}_{i}\right)}\right\} \cup \omega: i<n\right\}$ is not a Fréchet space as a subspace of the product $\prod\left\{F\left(\mathscr{Q}_{i}\right): i<n\right\}$ of Franklin compact spaces.

A space $X$ has countable tightness if for each $A \subset X$ and $x \in \operatorname{cl} A$, there is a countable subset $B$ of $A$ such that $x \in \operatorname{cl} B$.

Lemma 3 (V. I. Malyhin [3]). Suppose that $X_{i}$ is a compact space with countable tightness for each $i \in \omega$. Then the product $\Pi\left\{X_{i}: i \in \omega\right\}$ also has countable tightness.

G. Gruenhage constructed the following maximal almost disjoint family in order to show the existence of a countable Fréchet space $X$ such that $X^{n}$ is Fréchet for all $n \in \omega$, but $X^{\omega}$ is not Fréchet.

Lemma 4 [2] (MA). There is an infinite maximal almost disjoint family $\mathcal{I}=\bigcup\left\{\mathcal{J}_{i}: i \in \omega\right\}$ of infinite subsets of $\omega$ satisfying the following properties:

(a) $\mathcal{J}_{i} \cap \mathcal{G}_{j}=\varnothing$ if $i \neq j$;

(b) each finite subset of $\omega$ is contained in a member of $\mathcal{G}_{i}$ for each $i \in \omega ;$ and

(c) suppose that $k \in \omega, J \subset k,\left\{I_{j}: j \in J\right\} \subset \mathcal{G}$ and $A \subset\left(\prod\{\omega: i \in k-J\}\right)$ $\times\left(\prod\left\{I_{j}: j \in J\right\}\right)$ are such that

$$
A \cap\left[\left(\prod\{\omega-E(i): i \in k-J\}\right) \times\left(\prod\left\{I_{j}-F(j): j \in J\right\}\right)\right] \neq \varnothing
$$

whenever $E(i)$ is a finite union of members of $\mathcal{G}$, and $F(j)$ is a finite subset of $\omega$. Then for each $m \in \omega$, there is a sequence $\left\{a_{0}, a_{1}, \cdots\right\}$ in $A$ such that whenever $E(i)$ is a finite union of members of $\mathcal{G}-\mathcal{I}_{m}$ and $F(j)$ is a finite subset of $\omega$, there is a natural number $n$ satisfying that

$$
\left\{a_{n}, a_{n+1}, a_{n+2}, \cdots\right\} \subset\left(\prod\{\omega-E(i): i \in k-J\}\right) \times\left(\prod\left\{I_{j}-F(j): j \in J\right\}\right) .
$$

Proof (Sketch). We shall follow the construction of $\mathcal{G}=\bigcup\left\{\mathcal{G}_{i}: i \in \omega\right\}$ $=\left\{I_{i}(\alpha): \alpha<\mathrm{c}, i \in \omega\right\}$ and the notation in [2]. Obviously the property (a) holds. By the construction, for each $i \in \omega$ and a finite subset $F$ of $\omega$, there is $n \in \omega$ with $F \subset I_{i}(n) \in \mathcal{G}_{i}$. Therefore (b) is satisfied. It remains to check (c). Suppose the assumption of (c). Similarly to the last paragraph of the proof of Theorem in [2], take $\kappa<\mathfrak{c}, \beta_{0}=\beta(\kappa)$, the sequence $\vec{x}_{0}, \vec{x}_{1}, \cdots$ in $A=A_{\beta(x)}$ and the partition $\omega=\bigcup\left\{W_{m}: m \in \omega\right\}$ at the $\kappa$-stage. Define $\left\{a_{0}\right.$, $\left.a_{1}, \cdots\right\}=\left\{\vec{x}_{n}: n \in W_{m}\right\}$. Observe that $I_{m}(k)=\left\{\pi_{i}\left(\vec{x}_{n}\right): n \in W_{m}, i \in k-J\right\} \in \mathcal{J}_{m}$, and hence for each $I \in \mathcal{G}-\mathcal{G}_{m}, I \cap I_{m}(\kappa)$ is finite because $\mathcal{G}$ is an almost disjoint family. Now it is not difficult to check the property (c).

3. Example.

Lemma 5 (MA). For each $n$ with $3 \leqq n \leqq \omega$, there is a family $\left\{X_{i}: i<n\right\}$ of compact Fréchet spaces such that

(a) if $k<n$ and $\left\{Y_{i}: i<k\right\}$ is a family where each $Y_{i}$ is equal to $X_{j}$ for 
some $j<n$, then the product $\prod\left\{Y_{i}: i<k\right\}$ is Fréchet, but

(b) $\prod\left\{X_{i}: i<n\right\}$ is not Fréchet.

Proof. Let $3 \leqq n \leqq \omega$ and $\mathcal{I}=\bigcup\left\{\mathcal{G}_{i}: i \in \omega\right\}$ be the family in Lemma 4 . If $n<\omega$, define $\mathscr{Q}_{i}=\left\{I^{*}: I \in \mathcal{G}_{i}\right\}$ for each $i<n-1$ and $\mathscr{Q}_{n-1}=\left\{I^{*}: I \in \bigcup\left\{\mathcal{J}_{i}\right.\right.$ : $n-1 \leqq i \in \omega\}\}$. If $n=\omega$, simply define $\mathscr{L}_{i}=\left\{I^{*}: I \in \mathcal{J}_{i}\right\}$ for each $i<n=\omega$. Define $X_{i}=F\left(\mathscr{Q}_{i}\right)$ for each $i<n$. We will show that the family $\left\{X_{i}: i<n\right\}$ of Franklin compact spaces is the desired family. Since $\mathscr{Q}=\bigcup\left\{\mathscr{Q}_{i}: i<n\right\}$ is an infinite maximal disjoint family of clopen subsets of $\omega^{*}$, it follows from Lemma 2 that $\prod\left\{X_{i}: i<n\right\}$ is not Fréchet. So it remains to show the property (a) of our theorem.

Let $k<n$ and $\left\{Y_{i}: i<k\right\}$ be a family satisfying that each $Y_{i}$ is equal to $X_{j}$ for some $j<n$. We show that $Y=\prod\left\{Y_{i}: i<k\right\}$ is Fréchet. Since $k<n$, we can find $m<n$ such that $X_{m} \neq Y_{i}$ for each $i<k$. Fix such $m$. To show the Fréchet property of $Y$, let $y \in Y, B \subset Y$ and $y \in \operatorname{cl} B$. Without loss of generality, we may assume that $y=\left(\left\{I_{0}^{*}\right\},\left\{I_{1}^{*}\right\}, \cdots,\left\{I_{p-1}^{*}\right\}, \infty_{p}, \cdots, \infty_{p+q-1}\right.$, $\left.\infty_{p+q}, \cdots, \infty_{k-1}\right)$ and $B \subset \omega^{p} \times \mathcal{Q}_{p} \times \cdots \times \mathcal{Q}_{p+q-1} \times \omega^{k-p-q}$. The other cases are trivial or reducible to a case similar to this one.

Since every Franklin compact space has countable tightness, by Lemma 3, $Y$ also has countable tightness. So we may assume that $B$ is a countable set. Then there is a countable subfamily $Q_{p+r} \subset \mathscr{Q}_{p+r}$ for each $r=0,1,2, \cdots, q-1$ such that $B \subset \omega^{p} \times Q_{p} \times \cdots \times Q_{p+q-1} \times \omega^{k-p-q}$. Pick an arbitrary member $I_{p+r}^{*} \in \mathcal{Q}_{p+r}$ for each $r=0,1, \cdots, q-1$. Since $\left\{\infty_{p+r}\right\}$ $\cup Q_{p+r}$ is homeomorphic to a convergent sequence, it is homeomorphic to $\left\{I_{p+r}^{*}\right\} \cup I_{p+r} \subset\left\{I_{p+r}^{*}\right\} \cup \omega$. Hence we may assume moreover that $y=\left(\left\{I_{0}^{*}\right\},\left\{I_{1}^{*}\right\}\right.$, $\left.\cdots,\left\{I_{p-1}^{*}\right\},\left\{I_{p}^{*}\right\}, \cdots,\left\{I_{p+q-1}^{*}\right\}, \infty_{p+q}, \cdots, \infty_{k-1}\right)$ and $B \subset \omega^{p} \times \omega^{q} \times \omega^{k-p-q}=\omega^{k}$.

By the argument similar to [2], we obtain a set $J \subset k$, a family $\left\{I_{j}\right.$ : $j \in J\} \subset \mathcal{G}$ and a subset $A$ of $B$ satisfying the assumption of Lemma 4 (c). Recall that $X_{m} \neq Y_{i}$ for each $i<k$. Therefore the family of all sets of the form $\left(\prod\{\omega-E(i): i \in k-J\}\right) \times\left(\prod\left\{I_{j}-F(j): j \in J\right\}\right)$, where $E(i)$ is a finite union of members of $\mathcal{G}-\mathcal{J}_{m}$ and $F(j)$ is a finite subset of $\omega$, becomes a local network at $y$ in $Y$. Hence the sequence $\left\{a_{0}, a_{1}, \cdots\right\}$ in $A$ obtained by Lemma 4 (c) converges to $y$. The proof is completed.

Theorem 6 (MA). For each $n$ with $3 \leqq n \leqq \omega$, there is a compact Fréchet space $X$ such that $X^{k}$ is Fréchet for any $k<n$, but $X^{n}$ is not Fréchet.

Proof. Take the family $\left\{X_{i}: i<n\right\}$ of Lemma 5 . If $n<\omega$, let $X$ be the disjoint topological sum $\oplus\left\{X_{i}: i<n\right\}$. If $n=\omega$, let $X$ be the one point compactification of $\oplus\left\{X_{i}: i<n=\omega\right\}$. Since $X^{n}$ contains $\prod\left\{X_{i}: i<n\right\}, X^{n}$ is not Fréchet. It remains to prove that $X^{k}$ is Fréchet for any $k<n$. If $n<\omega$, this follows immediately from Lemma 5 .

Suppose $n=\omega$. Denote by $p$ the only one point of the set $X-\oplus\left\{X_{i}\right.$ : $i<\omega\}$. We must show the Fréchet property at points of $X^{k}$ whose coordinates contain $p$. But the neighborhood base at $p$ in $X$ is the same as a convergent sequence if we identify each $X_{i}$ to a point $x_{i}$. Therefore by the 
argument similar to the proof of Lemma 5, we can show that $X^{k}$ is Fréchet. Problem. Are Lemma 5 and Theorem 6 true within ZFC?

\section{References}

[1] T. K. Boehme and M. Rosenfeld: An example of two compact Hausdorff Fréchet spaces whose product is not Fréchet. J. London Math. Soc., 8, 339-344 (1974).

[2] G. Gruenhage: A note on the product of Fréchet spaces. Topology Proceedings, 3, 109-115 (1978).

[3] V. I. Malyhin: On tightness and Suslin number in $\exp X$ and in a product of spaces. Soviet Math. Dokl., 13, 496-499 (1972).

[4] V. I. Malyhin and B. E. Šapirovskiǐ: Martin's axiom and properties of topological spaces. ibid., 14, 1746-1751 (1973).

[5] E. Michael: A quintuple quotient quest. Gen. Topology Appl., 2, 91-138 (1972).

[6] T. Nogura: The product of $\left\langle\alpha_{i}\right\rangle$-spaces. Topology Appl., 21, 251-259 (1985).

[ 7 ] R. C. Olson: Bi-quotient maps, countably bi-sequential spaces, and related topics. Gen. Topology Appl., 4, 1-28 (1974).

[ 8 ] P. Simon: A compact Fréchet space whose square is not Fréchet. Comment. Math. Univ. Carolinae, 21, 749-753 (1980). 